Research Paper

\title{
Fluid intake-to-bed time, nocturia frequency and the risk of urothelial carcinoma of the bladder: a case-control study
}

\author{
Jianfeng Cui ${ }^{1}$, Qiyu Bo ${ }^{2}$, Ning Zhang ${ }^{1}$, Shouzhen Chen ${ }^{1}$, Meng $\mathrm{Yu}^{1}$, Shiyu Wang ${ }^{1}$, Jie Han ${ }^{3}$, Pengxiang \\ Chen ${ }^{4}$, Dongqing Zhang ${ }^{1}$, Yaofeng Zhu ${ }^{1 凶}$, Benkang Shi ${ }^{1 凶}$ \\ 1. Department of Urology, Qilu Hospital of Shandong University, Jinan, 250012 P.R. China; \\ 2. Department of First Operating Room, Qilu Hospital of Shandong University, Jinan, 250012 P.R. China; \\ 3. Department of Radiation Oncology, Shandong Cancer Hospital and Institute affiliated to Shandong University, Jinan, P.R. China; \\ 4. Department of Radiation Oncology, Qilu Hospital of Shandong University, Jinan, 250012 P.R. China. \\ $\triangle$ Corresponding authors: Benkang Shi and Yaofeng Zhu; E-mail: bkang68@sdu.edu.cn, yaofengzhu2016@126.com; Fax: +86-531-86927544; Tel: \\ $+86-18769780320$ \\ (c) Ivyspring International Publisher. This is an open access article distributed under the terms of the Creative Commons Attribution (CC BY-NC) license \\ (https://creativecommons.org/licenses/by-nc/4.0/). See http://ivyspring.com/terms for full terms and conditions.
}

Received: 2017.06.21; Accepted: 2017.08.29; Published: 2017.09.15

\begin{abstract}
Objectives: To clarify the potential role of fluid intake-to-bed time and nocturia frequency on bladder cancer risk in a hospital-based case-control study with Chinese people.

Materials and Methods: Four hundred and seven patients with histologically diagnosed bladder cancer and 400 matched controls were enrolled in this study. Odds ratios (ORs) and $95 \%$ confidence intervals $(\mathrm{Cls})$ were calculated using conditional logistic regression model.

Results: After adjustment for potential confounders, the fluid intake-to-bed time was associated with a decreased risk of $\mathrm{BCa}$, with an $\mathrm{OR}$ of $0.586(95 \% \mathrm{Cl}=0.375-0.916, \leq 1 \mathrm{~h})$ and $0.257(95 \% \mathrm{Cl}=$ $0.162-0.407,>1 \mathrm{~h}$ ). The adjusted $\mathrm{OR}$ of $\mathrm{BC}$ a for subjects with more nocturia frequency ( $\geq 2$ times) was $2.268(95 \% \mathrm{Cl}=1.481-3474)$, compared to those with no nocturia.

Conclusion: We suggested strong protective effect of long fluid intake-to-bed time on BCa risk, especially in ones with $\geq 2$ times nocturia frequency. These results provide evidence for identifying high-risk individuals and modifying their behaviors and lifestyle.
\end{abstract}

Key words: bladder cancer; case-control study; fluid intake-to-bed time; nocturia

\section{Introduction}

Bladder cancer (BCa) is the sixth most commonly diagnosed cancer in the male population worldwide [1] and 11th when both genders are considered [2] according to the latest published global cancer statistics. Among the urogenital system cancers, BCa ranked second both in incidence and mortality. Worldwide, the BCa age-standardised mortality rate (per 100000 person-years) was 3.2 for men versus 0.9 for women [1]. Tobacco smoking [3], high-risk occupational exposures, such as aromatic amines, benzidine and polycyclic aromatic hydrocarbons [4, 5], family history [6] are the most well-established risk factors for $\mathrm{BCa}$, and the risk among smokers is reported to be approximately 2 -fold to 6-fold that among non-smokers. In the developing world, particularly Africa and Western Asia, the increasing risk of $\mathrm{BCa}$ is associated with chronic infection with Schistosoma hematobium [7].

The "urogenous contact" hypothesis states that high fluid intake may reduce the exposure to potential carcinogens by diluting the urine and reducing the contact time with the bladder urothelium through increasing urination frequency, leading to decreased risk of $\mathrm{BCa}$ [8-11], and a meta-analysis suggested that high fluid intake might have a protective effect on $\mathrm{BCa}$ in Asian people [12]. But bladder wall extension 
associated with a high fluid intake or a low urination frequency might allow the carcinogens in the urine to come into contact with the deeper layers of the bladder urothelium, and increase the risk of BCa [13]. Therefore, studies focused on the fluid intake and risk of BCa didn't reach a consensus agreement.

Depended on the results from previous studies, we hypothesized that drinking water before going to bed could lead the potential carcinogens stay in the bladder and extend the bladder wall which could increase the contact time and proportion of bladder urothelium. To our knowledge, the risk of fluid intake-to-bed time in $\mathrm{BCa}$ has not been investigated yet. Thus, the aim of this study is to clarify the potential influence of fluid intake-to-bed time on $\mathrm{BCa}$ risk in a hospital-based case-control study with Chinese people.

\section{Material and Methods}

\section{Study design and study population}

421 newly cases of bladder cancer were recruited from the Qilu Hospital of Shandong University from March 2015 and March 2017. And 407 patients were histologically diagnosed with primary urothelial carcinoma, 14 patients were excluded because of being diagnosed with other types of $\mathrm{BCa}$, such as adenocarcinoma, squamous carcinoma and so on. And all $407 \mathrm{BCa}$ patients had confirmed without autoimmune disease or cancer in other systems at the time of recruitment. The control group included patients without smoking-related disease, cancer in other systems and autoimmune disease or healthy person who visited same hospital for annual health checkups, such as hernias, osteoarthritis and so on. No restrictions on age, sex, race or cancer stage in both groups. Total 400 cases were selected into control group at the same time and same hospital. This study was approved by the Institutional Ethics Committee of the Qilu Hospital of Shandong University, and all participants provided written informed consent.

\section{Questionnaire Data}

The experienced interviewers interviewed patients themselves or patients' relative for those who were not able to face illness objectively in case and control groups during hospitalization. The questionnaire included gender, age, body mass index (BMI), smoking status, education level, amount of total fluid intake, fluid type, fluid intake-to-bed time and nocturia frequency. BMI was defined according to the criteria recommended by the World Health Organization. The smoking status is performed by following the protocol of J Wang et al [14]. The fluid intake-to-bed time was defined as the most usual time (more than fifty percent of the days) interval from drinking more than $100 \mathrm{ml}$ fluid to going to bed in the past decade.

Then, the detailed collected information was categorized as follows, BMI was classified into thin $(<18.5)$, normal (18.5-24.9) and obesity (BMI $\geq 25)$, the amount of total fluid intake was divided into less than $1000,1000-2500$ and more than $2500 \mathrm{ml}$, fluid intake-to-bed time was classified into immediately, less than $1 \mathrm{~h}$ and more than $1 \mathrm{~h}$. And nocturia frequency was divided into 0,1 and more than 1 time.

\section{Statistical analysis}

Distributions of characteristics between cases and controls were tested using the $\chi^{2}$ test for categorical variables and paired student's t-test for continuous variables. Odds ratios (ORs) and 95\% confidence intervals (CIs) were calculated using conditional logistic regression models to estimate the relation between exposures and the risk of $\mathrm{BCa}$, and the analyses were adjusted for basic factors by using conditional multiple logistic regression models. Then, we further analyzed with the adjusted factors for the risk or protective factors for $\mathrm{BCa}$, including amount of total fluid intake and fluid type. The two-sided $p$ value was used in our analyses, and a $p$ value of less than 0.05 was considered statistically significant. Statistical analysis was performed using STATA version 13.0 (College Station, Texas, USA).

Table 1. Characteristics of cases and controls.

\begin{tabular}{|c|c|c|c|}
\hline Characteristics & Cases $(n=407)$ & Controls $(n=400)$ & $P$ value \\
\hline & $\mathrm{N}(\%)$ & $\mathrm{N}(\%)$ & \\
\hline Gender & & & 0.16 \\
\hline Male & $310(76.2)$ & $321(80.3)$ & \\
\hline Female & $97(23.8)$ & 79 (19.7) & \\
\hline Age (years) & & & 0.983 \\
\hline$\leq 40$ & $27(6.6)$ & $29(7.3)$ & \\
\hline $41-50$ & $49(12.0)$ & $44(11.0)$ & \\
\hline $51-60$ & 89 (21.9) & $91(22.8)$ & \\
\hline $61-70$ & $125(30.7)$ & $123(30.8)$ & \\
\hline$>70$ & 117 (28.7) & $113(28.3)$ & \\
\hline BMI $\left(\mathrm{kg} / \mathrm{m}^{2}\right)$ & & & 0.669 \\
\hline$<18.5$ & $12(2.9)$ & $11(2.8)$ & \\
\hline $18.5-24.9$ & $209(51.4)$ & $218(54.5)$ & \\
\hline$\geq 25$ & $186(45.7)$ & $171(42.8)$ & \\
\hline Smoking status & & & $<0.001$ \\
\hline Never & $154(37.8)$ & $241(60.3)$ & \\
\hline Former & $88(21.6)$ & $48(12.0)$ & \\
\hline Current & $165(40.5)$ & $111(27.8)$ & \\
\hline Education level & & & 0.919 \\
\hline None & $38(9.3)$ & $37(9.3)$ & \\
\hline Primary School & $175(43.0)$ & $172(43.0)$ & \\
\hline High School & $112(27.5)$ & 117 (29.3) & \\
\hline College & $82(20.1)$ & $74(18.5)$ & \\
\hline
\end{tabular}

\section{Results}

407 cases and 400 matched control subjects were enrolled in this study. Controls were accepted for the 
following reasons: healthy person (58\%), hernias $(21 \%)$, osteoarthritis $(12 \%)$, fractures $(5 \%)$ and dermatologic disorders (4\%). The baseline characteristics of cases and controls were shown in Table 1 . The cases and controls were similar with respect to the percentage in gender $(p=0.16)$, age $(p=0.983)$, BMI $(p=0.669)$ and education levels $(p=0.919)$, but the percentage of former smoker and current smoker was higher among $\mathrm{BCa}$ patients $(\mathrm{p}<0.001)$.

The risks of $\mathrm{BCa}$ in relation to type of fluid intake, amount of total fluid intake, fluid intake-to-bed time and nocturia frequency are shown in Table 2. After adjustment for potential confounders, for fluid type, the purified water and tea intake had no significant differences in reducing the risk of $\mathrm{BCa}$, compared to the boiled water $(\mathrm{OR}=0.671$, $95 \% \mathrm{CI}=0.406-1.109 ; \mathrm{OR}=1.213,95 \% \mathrm{CI}=0.865-1.702$, respectively). For total fluid intake, we observed a $45.3 \%$ decrease in risk for person consuming $1000-2500 \mathrm{ml} /$ day compared with those consuming
$<1000 \mathrm{ml} /$ day. The associations were similar when stratified on basic factors, with an OR of 0.545 (95\% $\mathrm{CI}=0.348-0.83$ ), but no significant difference was found in person consuming more than $2500 \mathrm{ml} /$ day. The fluid intake-to-bed time was associated with a decreased risk of $\mathrm{BCa}$, with an OR of $0.586(95 \% \mathrm{CI}=$ $0.375-0.916, \leq 1 \mathrm{~h})$ and 0.257 (95\% CI= 0.162-0.407, >1h). Risk was increased when person had 2 times and more than 2 times nocturia $(\mathrm{OR}=2.268,95 \% \mathrm{CI}=$ 1.481-3474).

We investigated the impact of fluid intake-to-bed time and nocturia frequency on the risk of $\mathrm{BCa}$ stratified by the smoking status (Table 3 ). The risk decreased with increasing fluid intake-to-bed time among never, former, and current smokers, although only more than $1 \mathrm{~h}$ was statistically significant (Table 3 ). And we observed a statistically decreased risk in never smokers $(\mathrm{OR}=4.028,95 \% \mathrm{CI}=2.038-7.960)$ for nocturia frequency, not in former smokers and current smokers.

Table 2. Risk of bladder cancer associated with fluid intake and nocturia frequency.

\begin{tabular}{|c|c|c|c|c|c|}
\hline & Case /Control & Crude OR $(95 \%$ CI) & $P$ value & Adjusted OR* $(95 \% \mathrm{CI})$ & $\mathrm{P}$ value \\
\hline \multicolumn{6}{|l|}{ Type of fluid intake } \\
\hline Boiled water & $248 / 241$ & 1 (reference) & & 1 (reference) & \\
\hline Purified water & $35 / 53$ & $0.567(0.36-0.892)$ & 0.014 & $0.671(0.406-1.109)$ & 0.12 \\
\hline Tea & $124 / 106$ & $1.217(0.885-1.673)$ & 0.226 & $1.127(0.802-1.583)$ & 0.263 \\
\hline \multicolumn{6}{|c|}{ Amount of total fluid intake } \\
\hline$<1000 \mathrm{ml} /$ day & $62 / 42$ & 1 (reference) & & 1 (reference) & \\
\hline $1000-2500 \mathrm{ml} /$ day & $256 / 317$ & $0.547(0.358-0.837)$ & 0.005 & $0.545(0.348-0.853)$ & 0.008 \\
\hline$>2500 \mathrm{ml} /$ day & $89 / 41$ & $1.470(0.858-2.52)$ & 0.161 & $1.351(0.767-2.378)$ & 0.297 \\
\hline \multicolumn{6}{|c|}{ Fluid intake-to-bed time } \\
\hline Immediately & $86 / 40$ & 1 (reference) & & 1 (reference) & \\
\hline$\leq 1 \mathrm{~h}$ & $203 / 162$ & $0.583(0.380-0.894)$ & 0.013 & $0.586(0.375-0.916)$ & 0.019 \\
\hline$>1 \mathrm{~h}$ & $118 / 198$ & $0.277(0.179-0.430)$ & $<0.001$ & $0.257(0.162-0.407)$ & $<0.001$ \\
\hline \multicolumn{6}{|l|}{ Nocturia frequency } \\
\hline 0 time & $67 / 83$ & 1 (reference) & & 1 (reference) & \\
\hline 1 time & $110 / 187$ & $0.729(0.489-1.086)$ & 0.12 & $0.762(0.498-1.166)$ & 0.211 \\
\hline$\geq 2$ times & $230 / 130$ & $2.192(1.488-3.228)$ & $<0.001$ & $2.268(1.481-3.474)$ & $<0.001$ \\
\hline
\end{tabular}

*Adjusted for gender, age, BMI, smoking status and education status

Table 3. Fluid intake and the risk of bladder cancer stratified by smoking status

\begin{tabular}{|c|c|c|c|c|c|c|c|c|c|}
\hline & \multicolumn{3}{|l|}{ Never smoker } & \multicolumn{3}{|l|}{ Former smoker } & \multicolumn{3}{|l|}{ Current smoker } \\
\hline & Case/Control & $\begin{array}{l}\text { Adjusted OR* } \\
(95 \% \mathrm{CI})\end{array}$ & $\mathrm{P}$ value & Case/Control & $\begin{array}{l}\text { Adjusted OR* } \\
(95 \% \mathrm{CI})\end{array}$ & $P$ value & Case/Control & $\begin{array}{l}\text { Adjusted OR* } \\
(95 \% \mathrm{CI})\end{array}$ & $P$ value \\
\hline \multicolumn{10}{|c|}{ Fluid intake-to-bed time } \\
\hline Immediately & $32 / 28$ & 1 (reference) & & $15 / 1$ & 1 (reference) & & $39 / 11$ & 1 (reference) & \\
\hline$\leq 1 \mathrm{~h}$ & $79 / 100$ & $\begin{array}{l}0.801 \\
(0.431-1.487)\end{array}$ & 0.482 & $48 / 20$ & $\begin{array}{l}0.132 \\
(0.012-1.447)\end{array}$ & 0.097 & $76 / 42$ & $\begin{array}{l}0.691 \\
(0.293-1.627)\end{array}$ & 0.398 \\
\hline$>1 \mathrm{~h}$ & $43 / 113$ & $\begin{array}{l}0.351 \\
(0.184-0.667)\end{array}$ & 0.001 & $25 / 27$ & $\begin{array}{l}0.044 \\
(0.004-0.523)\end{array}$ & 0.013 & $50 / 58$ & $\begin{array}{l}0.268 \\
(0.111-0.649)\end{array}$ & 0.004 \\
\hline \multicolumn{10}{|c|}{ Nocturia frequency } \\
\hline 0 time & $21 / 55$ & 1 (reference) & & $10 / 7$ & 1 (reference) & & $36 / 21$ & 1 (reference) & \\
\hline 1 time & $41 / 109$ & $\begin{array}{l}1.230 \\
(0.632-2.395)\end{array}$ & 0.542 & $24 / 20$ & $\begin{array}{l}0.514 \\
(0.118-2.232)\end{array}$ & 0.514 & $45 / 58$ & $\begin{array}{l}0.472 \\
(0.216-1.031)\end{array}$ & 0.06 \\
\hline$\geq 2$ times & $92 / 77$ & $\begin{array}{l}4.028 \\
(2.038-7.960)\end{array}$ & $<0.001$ & $54 / 21$ & $\begin{array}{l}1.060 \\
(0.267-4.203)\end{array}$ & 0.934 & $84 / 32$ & $\begin{array}{l}1.211 \\
(0.553-2.653)\end{array}$ & 0.632 \\
\hline
\end{tabular}

*Adjusted for gender, age, BMI, education status, type of fluid and amount of fluid 
Table 4. Adjusted odds ratios of BCa for fluid intake-to-bed time according to amount of total fluid intake and nocturia frequency

\begin{tabular}{|c|c|c|c|c|c|}
\hline \multirow[t]{2}{*}{ Fluid intake-to-bed time } & \multirow{2}{*}{$\begin{array}{l}\text { Immediately } \\
\text { Adjusted OR* }(95 \% \mathrm{CI})\end{array}$} & \multicolumn{2}{|l|}{$\leq 1 \mathrm{~h}$} & \multicolumn{2}{|l|}{$>1 \mathrm{~h}$} \\
\hline & & Adjusted OR* $(95 \% \mathrm{CI})$ & $\mathrm{P}$ value & Adjusted OR* $(95 \% \mathrm{CI})$ & P value \\
\hline \multicolumn{6}{|c|}{ Amount of total fluid intake } \\
\hline$<1000 \mathrm{ml} /$ day & 1 (reference) & $0.257(0.06-1.108)$ & 0.068 & $0.374(0.093-1.510)$ & 0.167 \\
\hline $1000-2500 \mathrm{ml} /$ day & 1 (reference) & $1.064(0.618-1.830)$ & 0.824 & $0.376(0.214-0.658)$ & 0.001 \\
\hline$>2500 \mathrm{ml} /$ day & 1 (reference) & $0.028(0.003-0.259)$ & 0.002 & $0.012(0.001-0.154)$ & 0.001 \\
\hline \multicolumn{6}{|l|}{ Nocturia frequency } \\
\hline 0 time & 1 (reference) & $0.087(0.017-0.454)$ & 0.004 & $0.036(0.007-0.199)$ & $<0.001$ \\
\hline 1 time & 1 (reference) & $0.711(0.340-1.485)$ & 0.364 & $0.432(0.199-0.937)$ & 0.034 \\
\hline$\geq 2$ times & 1 (reference) & $0.827(0.399-1.713)$ & 0.608 & $0.244(0.117-0.509)$ & $<0.001$ \\
\hline
\end{tabular}

*Adjusted for gender, age, BMI, smoking status, education level and type of fluid

Then we further explored the influence of fluid intake-to-bed time on the risk of $\mathrm{BCa}$ stratified according to the amount of total fluid intake and nocturia frequency (Table 4). When subjects were analyzed according to the amount of total fluid intake, the adjusted OR of BCa for long fluid intake-to-bed time relative to short fluid intake-to-bed time was lower for $>2500 \mathrm{ml} /$ day subjects (adjusted $\mathrm{OR}=0.012$, $95 \% \mathrm{CI}=0.001-0.154)$ than $<1000 \mathrm{ml} /$ day subjects (adjusted $\mathrm{OR}=0.374, \quad 95 \% \mathrm{CI}=0.093-1.510$ ). When subjects were analyzed according to the nocturia frequency, the adjusted $\mathrm{OR}$ of $\mathrm{BCa}$ for long fluid intake-to-bed time relative to short fluid intake-to-bed time was both statistically significant in 0 time nocturia subjects (adjusted OR=0.036, $95 \% \mathrm{CI}=$ $0.007-0.199$ ) and $\geq 2$ times subjects (adjusted OR= $0.244,95 \% \mathrm{CI}=0.117-0.509$ ), but the positive effect of longer fluid intake-to-bed time had been impaired by more nocturia frequency. And we also found that subjects who had $\geq 1$ time nocturia, fluid intake-to-bed time $(\leq 1 \mathrm{~h})$ was no longer a protective factor $(\mathrm{OR}=$ $0.711,95 \% \mathrm{CI}=0.340-1.485 ; \mathrm{OR}=0.827,95 \% \mathrm{CI}=$ $0.399-1.713$, respectively).

\section{Discussion}

To our knowledge, this is the first study evaluating the relationship of fluid intake-to-bed time, nocturia frequency and the risk of $\mathrm{BCa}$. In this case-control study, we observed longer fluid intake-to-bed time and less nocturia frequency were associated with a reduced risk of $\mathrm{BCa}$. The results were similar when stratified by smoking status, but the low nocturia frequency in former smokers couldn't reflect the reduced risk of BCa. Moreover, we found the beneficial effects of long fluid intake-to-bed time in the subjects with high nocturia frequency.

The origin of $\mathrm{BCa}$ is still obscure. The "urogenous contact" hypothesis was first proposed by Oyasu, Hopp [10] and Melicow [15], which attributed the growth of $\mathrm{BCa}$ to the prolonged exposure to potential carcinogens in urine. An experiment data showed that separating part of bladder in dogs by surgery to prevent from contacting urine, then dealt dogs with $\beta$-betanaphthyl amine, finally bladder which contacted urine generated the $\mathrm{BCa}$, not in the separating part [9]. And short fluid intake-to-bed time could increase the amount of urine in bladder, accumulate more potential carcinogens, extent the contact area of bladder wall and urine, may introduce the carcinogens to the deeper layer of the bladder urothelium [12], and finally increase the risk of BCa. The results in our study were consistent with the "urogenous contact" hypothesis.

Frequency of urination is directly related to the intensity and duration of urothelium distension [16]. Several studies investigated the role of frequency of urination in the etiology of $\mathrm{BCa}[8,16-18]$, but only few studies explored the association for nocturia frequency and BCa risk. Our study found the positive correlation for more than 1 time nocturia and $\mathrm{BCa}$ $(\mathrm{OR}=2.268 ; 95 \% \mathrm{CI}=1.481-3.474 ; \mathrm{p}<0.001)$, not for 1 time $(\mathrm{OR}=0.762 ; 95 \% \mathrm{CI}=0.498-1.166 ; \mathrm{p}=0.211)$. One study [18] showed that an inverse trend in BCa risk with increasing number of nocturia. According to the definition of International Continence Society (ICS) in 2002 [19], nocturia is the complaint that the subject has to wake up at night 1 or more times to void, each void is preceded and followed by sleep. And nocturia, especially two or more times per night, is associated with a damaged health-related quality of life [20,21], such as depressive symptoms, sleep and et al. Madhu et al [22] found that prevalence of BCa increased with increasing episodes of nocturia which was consistent with our study.

We observed moderate total fluid intake could significantly reduce the risk of $\mathrm{BCa}$ in the present study, compared to the small amount of fluid intake, and high amount of total fluid intake was likely to increase the risk of $\mathrm{BCa}$, but not reached the statistical difference. This was consistent with the hypothesis which declared that moderate fluid intake might dilute potiential carcinogens in the urine and increase the voiding frequency, thus reducing contact of carcinogens with the bladder wall $[23,24]$. However, high total fluid intake might increase the net flow of potential carcinogens into the bladder and finally 
increase the risk of $\mathrm{BCa}$ [13]. And this hypothesis was verified by some studies [14, 23, 25], but some studies [4, 16, 26] detected high total fluid intake was associated with the decreased risk of BCa. Also, several studies [27-29] found no association with decreased risk. The inconsistent results might be caused by varies calculation of total fluid intake and types of fluid. Our study found tea intake had no association with decreased risk of $\mathrm{BCa}$, compared to the boiled water intake, which was inconsistent with the result of Wang et al [14]. And we have doubt about this result, for it is hard to explain and is not consistent with the protective role of tea.

Of the 7 million deaths from cancer in 2001 worldwide, an estimated 2.43 million were attributable to 9 potentially behavioral and environmental risk factors [30]. Modifying healthy lifestyle and environmental risk factors may greatly reduce the burden of cancers. Our study declared new potential risk factors of $\mathrm{BCa}$, shorter fluid intake-to-bed time and more nocturia frequency. We suggested to have moderate fluid intake and drinking last fluid at least $1 \mathrm{~h}$ before going to bed every day, and try to avoid $\geq 2$ times nocturia, and these may be helpful for preventing BCa. However, large-scale, different regions, different races and prospective studies are still needed to further testify whether fluid intake-to-bed time and nocturia frequency as risk factors of BCa.

In summary, our study showed shorter fluid intake-to-bed time and more nocturia frequency were inversely associated with the risk of $\mathrm{BCa}$, and regardless of how nocturia is defined, it is not a benign symptom. And high amount of fluid intake couldn't reduce the risk of $\mathrm{BCa}$, we suggest a protective effect of long fluid intake-to-bed time on $\mathrm{BCa}$ risk, especially in ones with $\geq 2$ times nocturia frequency.

\section{Abbreviations}

BCa: bladder cancer; BMI: body mass index; OR: odds ratio; $\mathrm{CI}$ : confidence interval

\section{Acknowledgements}

This work was supported by the National Natural Science Foundation of China (Grant 81470987 and 81670687 to B. Shi ), the Tai Shan Scholar Foundation to B. Shi, the Science and Technology Development Project of Jinan (Grant 201602155 to B. Shi), Natural Science Foundation of Shandong Province (Grant ZR2014HQ062 to Y. Zhu) and Science Foundation of Qilu Hospital of Shandong University (Grant 2015QLMS28 to B. Shi; Grant 2015QLQN21 to Y. Zhu ), Medicine and Health Science Technology Development Project of Shandong Province (Grant
2014WS0138 to Y. Zhu), Primary Research and Developement Plan of Shandong Province (Grant 2016GSF201011 to D. Zhang and 2016GSF201036 to Y. $\mathrm{Zhu})$.

\section{Competing Interests}

The authors have declared that no competing interest exists.

\section{References}

[1] Torre LA, Bray F, Siegel RL, et al. Global cancer statistics, 2012. CA Cancer J Clin. 2015; 65:87-108

[2] Babjuk M, Bohle A, Burger M, et al. EAU Guidelines on Non-Muscle-invasive Urothelial Carcinoma of the Bladder: Update 2016. Eur Urol. 2016; 71: 447-61

[3] Parkin DM. The global burden of urinary bladder cancer. Scand J Urol Nephrol Suppl. 2008; 12-20

[4] Michaud DS, Kogevinas M, Cantor KP, et al. Total fluid and water consumption and the joint effect of exposure to disinfection by-products on risk of bladder cancer. Environ Health Perspect. 2007; 115:1569-72

[5] Olfert SM, Felknor SA, Delclos GL. An updated review of the literature: risk factors for bladder cancer with focus on occupational exposures. South Med J. 2006; 99:1256-63

[6] Aben KK, Witjes JA, Schoenberg MP, et al. Familial aggregation of urothelial cell carcinoma. Int J Cancer. 2002; 98:274-8

[7] Parkin DM. The global health burden of infection-associated cancers in the year 2002. Int J Cancer. 2006; 118:3030-44

[8] Braver DJ, Modan M, Chetrit A, et al. Drinking, micturition habits, and urine concentration as potential risk factors in urinary bladder cancer. J Natl Cancer Inst. 1987; 78:437-40

[9] Mcdonald DF, Lund RR. The role of the urine in vesical neoplasm. I Experimental confirmation of the urogenous theory of pathogenesis. J Urol. 1954; 71:560-70

[10] Oyasu R, Hopp ML. The etiology of cancer of the bladder. Surg Gynecol Obstet. 1974; 138:97-108

[11] Malats N, Real FX. Epidemiology of bladder cancer. Hematol Oncol Clin North Am. 2015; 29:177-89

[12] Bai Y, Yuan H, Li J, et al. Relationship between bladder cancer and total fluid intake: a meta-analysis of epidemiological evidence. World J Surg Oncol. 2014; $12: 223$

[13] Claude J, Kunze E, Frentzel-Beyme R, et al. Life-style and occupational risk factors in cancer of the lower urinary tract. Am J Epidemiol. 1986; 124:578-89

[14] Wang J, Wu X, Kamat A, et al. Fluid intake, genetic variants of UDP-glucuronosyltransferases, and bladder cancer risk. Br J Cancer. 2013; 108:2372-80

[15] Melicow MM. Tumors of the bladder: a multifaceted problem. J Urol. 1974; 112:467-78

[16] Jiang X, Castelao JE, Groshen S, et al. Water intake and bladder cancer risk in Los Angeles County. Int J Cancer. 2008; 123:1649-56

[17] Radosavljevic V, Jankovic S, Marinkovic J, et al. Fluid intake and bladder cancer. A case control study. Neoplasma. 2003; 50:234-8

[18] Silverman DT, Alguacil J, Rothman N, et al. Does increased urination frequency protect against bladder cancer? Int J Cancer. 2008; 123:1644-8

[19] van Kerrebroeck P, Abrams P, Chaikin D, et al. The standardisation of terminology in nocturia: report from the Standardisation Sub-committee of the International Continence Society. Neurourol Urodyn. 2002; 21:179-83

[20] Tikkinen KA, Johnson TN, Tammela TL, et al. Nocturia frequency, bother, and quality of life: how often is too often? A population-based study in Finland. Eur Urol. 2010; 57:488-96

[21] Coyne KS, Zhou Z, Bhattacharyya SK, et al. The prevalence of nocturia and its effect on health-related quality of life and sleep in a community sample in the USA. Bju Int. 2003; 92:948-54

[22] Madhu C, Coyne K, Hashim H, et al. Nocturia: risk factors and associated comorbidities; findings from the EpiLUTS study. Int J Clin Pract. 2015; 69:1508-16

[23] Bruemmer B, White E, Vaughan TL, et al. Fluid intake and the incidence of bladder cancer among middle-aged men and women in a three-county area of western Washington. Nutr Cancer. 1997; 29:163-8

[24] Pelucchi C, Bosetti C, Negri E, et al. Mechanisms of disease: The epidemiology of bladder cancer. Nat Clin Pract Urol. 2006; 3:327-40

[25] Vena JE, Graham S, Freudenheim J, et al. Drinking water, fluid intake, and bladder cancer in western New York. Arch Environ Health. 1993; 48:191-8

[26] Zhou J, Kelsey KT, Giovannucci E, et al. Fluid intake and risk of bladder cancer in the Nurses' Health Studies. Int J Cancer. 2014; 135:1229-37

[27] Ros MM, Bas BH, Buchner FL, et al. Fluid intake and the risk of urothelial cell carcinomas in the European Prospective Investigation into Cancer and Nutrition (EPIC). Int J Cancer. 2011; 128:2695-708

[28] Brinkman M, Zeegers MP. Nutrition, total fluid and bladder cancer. Scand J Urol Nephrol Suppl. 2008; 25-36 
[29] Geoffroy-Perez B, Cordier S. Fluid consumption and the risk of bladder cancer: results of a multicenter case-control study. Int J Cancer. 2001; 93:880-7

[30] Danaei G, Vander HS, Lopez AD, et al. Causes of cancer in the world:

comparative risk assessment of nine behavioural and environmental risk factors. Lancet. 2005; 366:1784-93 